\section{PROTECTING KIDS AROUND LIQUID LAUNDRY PACKETS: A NATIONAL EFFORT TO EDUCATE COMMUNITES}

Kate Carr, Torine Creppy. Safe Kids Worldwide, USA

\subsection{6/injuryprev-2016-042156.909}

Background Exposure to liquid laundry detergent packets is an emerging risk for young children in the United States. In 2014, poison control centres in the U.S. received 11,711 calls about children 5 and younger having been exposed to laundry detergent packets. This is an 11\% increase in calls from 2013, and an $85 \%$ increase from 2012. In a study of calls to U.S. poison control centres from 2012 and 2013, more than 700 children under six years were hospitalised and experienced serious effects as a result of exposure to laundry detergent packets. The number of exposures was greatest among 1- and 2-year-olds. According to the United States Consumer Product Safety Commission (CPSC), children can experience loss of consciousness, difficulty breathing, excessive vomiting, severe eye burns, and temporary vision loss.

Methods Safe Kids conducted a review of existing data and recommendations from the CPSC and the American Association of Poison Control Centres to inform the development of a national awareness campaign and educational materials for caregivers of young children.

Results The campaign garnered a total of 489 million impressions from traditional media and digital activation. We provide 400 Safe Kids' coalitions and more than 100 national public health and safety partners with access to bilingual educational materials and digital activation tools. We incorporated key safety messages into 180 local events reaching 125,000 people.

Conclusions The statistics are alarming, and there are global concerns as well. Changes to product design and packaging, including the addition of warning labels, are underway in many countries. Educational and awareness efforts in several countries are raising mindfulness among caregivers of this emerging risk to younger children. Safe Kids is exploring opportunities to extend education and outreach efforts in the United Kingdom, Italy, Spain, Canada, Japan, Israel, Austria, France and Poland.

\section{CASE-CONTROL STUDY OF INJURY INTERVENTION FOR PRESCHOOL CHILDREN IN HENGGANG, SHENZHEN}

${ }^{1}$ Hong Wang, ${ }^{2}$ Xiao-Xian Liu, 'Zhong-Huang Peng, ${ }^{3}$ Jiu-Zhong Liao, ${ }^{4}$ Feng Liu. ${ }^{1}$ Maternal and Child Health Hospital of Shenzhen City, Nanfang Medical University, Shenzhen City, Guangdong 518028, China; '2Department of Women and Children's Health, Tongji Medical College, Huazhong University of Science and Technology, Wuhan, Hubei 430030, China; ${ }^{3}$ People Hospital of Henggang Town, Shenzhen City, Guangdong 518000, China; ${ }^{4}$ Prevention and Health Care Institution of Henggang Town, Shenzhen City, Guangdong 518000, China

\subsection{6/injuryprev-2016-042156.910}

Background To explore effective interventions for child accidental injury prevention and to reduce the incidence of injury.

Methods Cluster random sampling method was adopted, and children in 19 kindergartens in Henggang, Shenzhen and their parents were selected as the objects of study. Nineteen kindergartens were randomly divided into intervention group and control group to carry out the injury intervention case-control study.
Results through a series of interventions, there were certain effects. After the end of the project, the injury incidence rates of the intervention group and the control group were $4.91 \%$, $10.64 \%$, and the difference was significant; the average costs of treatment for injuries of the intervention group and the control group were 168.4 Yuan and 206.8 Yuan, and the difference was statistically significant; compared with prior to the implementation of the project, the rate of various types of injuries after the end of the project declined, in which, the rate of mechanical injury, pet bites, accidental falls, burns and traffic accidents decreased significantly. The differences were significant.

Conclusions Injury interventions can effectively prevent and control the occurrence of injury.

Funding Shenzhen Medical Research Fund (No: 201102096 )

\section{THE 'CODE OF CARING' OPERATIONAL MODEL SUPPORTS CHILDREN AND YOUNG PEOPLE}

${ }^{11}$ Minna Koivuluoma, ${ }^{2}$ Sami Perälä, ${ }^{3}$ Tiina Pekkanen, ${ }^{1} J o h a n n a$ Sorvettula, ${ }^{4}$ Tuija Leppäkoski, ${ }^{4}$ Eija Paavilainen. ${ }^{1}$ South Ostrobothnia Health Care District; ${ }^{2}$ South Ostrobothnia Health Technology Development Centre; ${ }^{3}$ City of Seinäjoki; ${ }^{4}$ University of Tampere, Finland

\subsection{6/injuryprev-2016-042156.911}

Background Families' ill-health and aggravated problems have a direct impact on the well-being and safety of children and young people. Reactions to life's dilemmas tend to appear only after a delay, with families attempting to cope with problems on their own. Furthermore, service provision is strongly focused on remedial services.

Methods Under an operational model called 'the Code of caring', children and young people and their families may request aid in coping with everyday problems. Authorities and third-sector actors also have the option of submitting requests for cooperation in order to support or aid a young person whenever they encounter nascent problems. Requests for cooperation submitted online are forwarded to Youth outreach work, the representatives of which assess the situation together with the child, young person and family, helping them to access the services they need.

Results The 'Code of caring' operational model and an electronic communications system are operational in the town of Seinäjoki. The voluntary nature of receiving support and the way Youth outreach work conducts it work have proved meaningful for children, young people and their families. Collaboration by Youth outreach work with the various professionals has contributed positively to collaboration addressing the concerns of children and young people, including the principle of offering services from a single service counter. The problems of children and young people addressed have been related to mental health issues, intoxicants, crises in the family, study, work, training, as well as the skills in managing personal economy and everyday life. The results of the operational model will be presented at the Safety 2016 World Conference.

Conclusions The 'Code of caring' operational model and the electronic communications system have proved highly functional methods, seeking to promote children's and young people's safety and life management skills through early supportive measures. 


\section{MULTI-SECTORAL MODEL FOR CHECKING HEALTH, SAFETY AND COMMUNITY WELL-BEING IN SCHOOLS}

${ }^{1}$ Ulla Korpilahti, ${ }^{1}$ Marke Hietanen-Peltola, ${ }^{1}$ Heidi Peltonen, ${ }^{2,3}$ Riina Länsikallio, ${ }^{4}$ Tarja Hartikainen, ${ }^{5}$ Sari Wuolijoki, ${ }^{6}$ Marjaana Pelkonen. ${ }^{1}$ National Institute for Health and Welfare; ${ }^{2}$ Finnish National Board of Education; ${ }^{3}$ Trade Union of Education in Finland; ${ }^{4}$ Association of Finnish Local and Regional Authorities; ${ }^{5}$ Finnish Institute of Occupational Health; ${ }^{6}$ Ministry of Social Affairs and Health

\subsection{6/injuryprev-2016-042156.912}

Background The Health Care Act (1326/2010, sections 16-17) prescribes that school and student health services must carry out triennial checks on the health and safety of school environments and well-being in learning communities in co-operation both with various authorities and with pupils, students and guardians. National follow-up data show, however, that checks have not been made comprehensively and that national guidance is needed.

Objective In 2013 preparations were started for a handbook and a model for the checks in order to promote health, safety and community well-being as well as multi-sectoral co-operation in schools and educational institutions. The steering group had representatives from the Ministry of Social Affairs and Health, the National Institute for Health and Welfare, the Finnish National Board of Education, the Finnish Institute of Occupational Health, the Association of Finnish Local and Regional Authorities and the Trade Union of Education in Finland. During the process the group received valuable feedback from nearly 40 experts, and the handbook and the check forms were piloted in six schools.

Results The handbook presents a new multi-sectoral model for checking the health, safety and community well-being in schools, covering the different stages of the checks: planning, implementation, monitoring, documentation, and communication of the results. It also includes the necessary forms for the checks and defines the roles of different actors. The handbook was published in autumn 2015.

Conclusions Multi-sectoral co-operation increases the health, safety and community well-being in schools and educational institutions as well as improves effectiveness, bringing savings and benefits to all parties. The handbook is primarily intended for national authorities, but it can also be used by pupils and students, by teachers and educators in different educational institutions, as well as by employees and other stakeholders in the relevant sectors.

\section{SPORT INJURIES IN CHILDREN: 10-YEAR EXPERIENCE AT THE RED CROSS WAR MEMORIAL CHILDREN'S HOSPITAL}

${ }^{1}$ Haiko K Jahn, ${ }^{2}$ Tamsin Carter-Smith, Jodie H Frost, ${ }^{2}$ Sebastian Van As. ${ }^{1}$ Royal Belfast Hospital for Sick Children, Belfast, Northern Ireland; ${ }^{2}$ Red Cross War Memorial Children's Hospital, Cape Town, South Africa; ${ }^{3}$ Great Ormond Street Hospital, London, England

\subsection{6/injuryprev-2016-042156.913}

Background Our review focuses on the incidence, aetiology and management of sports injuries in the paediatric population in a tertiary paediatric trauma unit in a developing country.

Methods The data from Trauma Unit Register at the Red Cross War Memorial Children's Hospital for the 10-year period from 2000 until 2010 was retrospectively analysed for all children under the age of 13-years, who were documented as having sustained any injury in a Sport setting.
Results Over the 10-year period 950 children (average age 9 years), who were presented to the trauma unit had sports related injuries. The male to female ratio was 5.6:1 (males 805, females 145). Whilst 212 needed immediate admission 738 were discharged on initial presentation. Abbreviated injury score was minor for 547, moderate for 393 and severe for 10 children.

The cause of injury was undefined falls in 416 and falls involving playground equipment in 87 cases. The site of injury was the upper limb 392 (forearm 120, elbow supracondylar 44, elbow other 60 , hand 82, wrist 74, upper arm 12), the lower limb 224 (tibia and fibula shaft and calf 70, foot 62, ankle 54, femur shaft and thigh, 29 hip and femoral neck 9) and the head 137 (scalp 41 , skull 41, brain closed 20, concussion 35 ) cases.

Fractures were the most common pathology (closed 416, open 10, fracture dislocation 7) followed by closed tissue injures 168 , abrasions 88 and lacerations in 67 cases.

Conclusions This review shows a male predominance in sport related injuries in the under 13-year old age group, which may reflect greater sporting participation by boys than girls in South Africa. The upper limb was the most commonly injured extremity and falls the most common cause of injury. This may be a result of a bracing type injury. Increasing sport participation by girls may change this injury pattern. Injury and fall prevention may decrease injuries in this age group.

\section{RISK FACTORS FOR ROAD TRAFFIC INJURIES AMONG SCHOOL CHILDREN IN RURAL BANGLADESH}

${ }^{1}$ Baset Md Kamran ul, ${ }^{2}$ Towner Elizabeth, Noor Tajkera, ${ }^{1}$ Rahman Aminur, ${ }^{1}$ Mashreky Saidur Rahman, ${ }^{1}$ Rahman AKM Fazlur. 'Centre for Injury Prevention and Research, Bangladesh (CIPRB); ${ }^{2}$ University of the West of England

\subsection{6/injuryprev-2016-042156.914}

Introduction Road traffic injuries (RTIs) are a major health problem internationally, causing illness, deaths and disabilities among young people. Research on RTIs in developing countries has been limited. We studied RTIs among primary school students in a rural area of Bangladesh.

Objective The objective of the study is to investigate risk factors for RTIs in school children.

Method We surveyed 778 grade IV and V students (aged 8-10 years) in Raiganj using a survey questionnaire. The survey was conducted at fifteen randomly selected primary schools with the cooperation of teachers and field supervisors. The questionnaire gathered data on socio-demographics, school journey, and knowledge $\&$ practice of road use.

Result The prevalence of risk factors (and confidence intervals) for road traffic injury among school children were estimated. The results from the 778 interviews were: $93.2 \%$ and $91.2 \%$ reported that they walked to and home from school; $52.2 \%$ of them were alone or accompanied by peers; $59.4 \%$ reported crossing busy roads or high way during their school journey ; $42.3 \%$ reported they had received no training regarding road use; and $26.4 \%$ re reported that they had RTIs in the last 12 months and most of these incidents occurrence were on the school journey (after school) when they were crossing or walking on the side of the road.

Conclusion The results are in accordance with the high rates of RTIs and huge exposure of risk factors among school children, reinforcing the need of integrated inter-sectoral actions, including training and awareness among school children. 